\title{
The Influence of Composite Polymeric Materials Topology Over the Shearing Modulus Using Virtual Instruments
}

\begin{abstract}
MARINELA MARINESCU, LARISA BUTU*, CLAUDIA BORDA, DELICIA ARSENE, MIHAI BUTU
Politehnica University of Bucharest, 313 Splaiul Independentei, 060042, Bucharest, Romania

This study presents research regarding the calculation of the mechanical characteristics of composite polymeric materials. By using LabVIEW' software a virtual instrument was created used for monitoring in real time the process of cross-linking the composite polymeric materials. The experiments were realized based on composite materials containing epoxy/fiberglass resin of different topologies. By means of the virtual instrument and of a sensor created based on the mechanical impedance analysis, implanted in the composite material, it was determined the $G$ shearing module of the composite material at different temperatures.
\end{abstract}

Keywords: composite polymeric materials, shearing module, sensor, virtual instrument

The rheological characteristics of the heat resistant resins, used as matrices of the composite materials, have a strong influence over the final mechanical properties of the manufactured pieces. The treatment, contextualizedmeaning processing the thermosetting polymers- refers to the variations of chemical and physical nature that are produced during the transformation of the unwrought material into its final rigid, infusible shape. During the treatment, the polymerization and the chemical bonds transform the polymer from a viscoelastic fluid into a gel and then into a glassy viscoelastic material [1]. The chemical process of cross-linking of an epoxy resin starts with the formation and development of a linear molecular structure of the chemical chain, which eventually transforms into a branched structure, and the laststep being obtaining three-dimensional connections between the previously formed branches. Once the chemical reaction starts the molecular mass grows rapidly and the formed chemical chains bond with one another and form a network with great molecular mass that increases as the process continues. This sudden and irreversible transformation from a viscous liquid state into an elastic gel characterized by a seeming infinite network is defined by the gelling point [2]. The viscosity of the resin begins to increase due to molecular connections $[3,4]$. When the gelling point is reached the average molecular mass $\mathrm{M}_{w}$ and the viscosity become infinite, indicating the shift from a viscoelastic liquid to a viscoelastic solid. After the gelling process, the vitrifying process of the formed chains follows. This process is characterized by a very important parameter namedtemperature of vitreous transition - T. In this state, the cross-linking of the resin is an extremelys slow process and from a practical point of view the vitrifying process puts a sudden end to it. To ensure the stability of the system resinthickener at higher temperatures than the treatment temperature the resins are currently afterwards treated at higher temperatures than $\mathrm{T}_{\text {, }}$ after the reaction between the resin and the thickener is completely finished [5].

Due to the variations in a large range of the rheological properties planning a single instrument capable to trace the rheological behavior during the whole cross-linking process is fairly difficult [6]. In this study, it is displayed an experimental stand that aided in the determination of the rheological measurements of the composite polymeric materials during their processing.

\section{Theoretical aspects}

Regarding the determination of the shearing module of the composite material it was necessary the mathematical modelling in the frequencies domain of the composite polymeric material system based on finding the response capacity. The real structure which has infinite degrees of liberty was replaced by the mathematical model of the linear dynamic system that has limited degrees of liberty, with constant parameters. At every resonance, the frequency was measured and the form of the vibration mode was determined.

The response of the system depends on both the characteristics of the mode of excitation of the sensor but also on the dynamic properties of the system. The studied system was considered a linear system, in which the input was noted with $x(t)$ meaning the excitation of the system, and the output $y(t)$ represents the answer. The response capacity in the frequency $H(\omega)$ of the system is given by the mathematical relationship:

$$
H(\omega)=\frac{Y(\omega)}{X(\omega)}
$$

where: $X(\omega)$ and $Y(\omega)$ are the Fourier transform of the input and output calculated with these mathematical relationships:

$$
\begin{aligned}
& X(\omega)=\int_{-\infty}^{+\infty} x(t) e^{-i \omega t} d t \\
& Y(\omega)=\int_{-\infty}^{+\infty} y(t) e^{-i \omega t} d t
\end{aligned}
$$

The sensor used in these experiments has these measurements: $b$ - the thickness $(0.15 \mathrm{~mm}), L$ - the length in contact with the resin $(60 \mathrm{~mm})$ and $I$ - the width $(6$ $\mathrm{mm})$. The speed $v(\omega)$ or the acceleration $a(\omega)$ were measured in the direction of the excitement force of the sensor (where $\omega$ is the angular frequency of the exciting force). In this case, the output's signals are the amplitude of the commuting sensor that vibrates inside the composite material and the phase angle between the exciting force and the measured acceleration.

For the geometry in the figure 1 in which the resin is cross-linked between the sensor and the layers of 


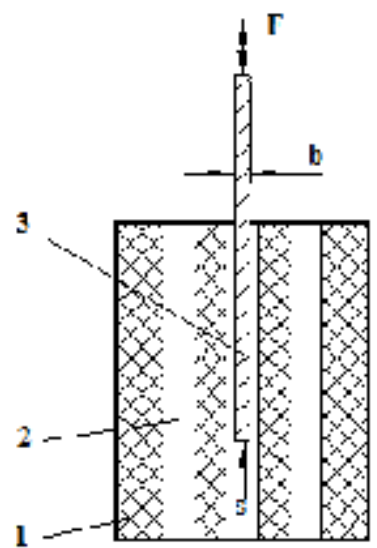

Fig. 1. Cross section of the area where the sensor is located: 1 - reinforcement fiber,

2 -the resin layer; 3 -sensor; b -sensor thickness; $F$-force; $\mathrm{S}$-shear force

reinforcement fibers, the complex shearing modulus $G^{*}(\omega)$ has the form:

$$
G^{*}=G^{\prime}+i G^{\prime \prime}
$$

where: $G^{\prime}$ is the load modulus; $G^{\prime \prime}$ the loss modulus.

Therefore, the studied system can be simplified according to the scheme from the figure 2 . In all the experiments the exciting force applied over the structure and the resulted movement were measured on the same surface. Additionally, for all the analyses the following approximations were made: the whole sistem treating resin-fibers can be represented as a dynamic linear system; the sensor used for monitoring the composite material moves between two layers of resin; the resin layer's thickness, that exists on both sides of the sensor $(\mathrm{h})$, between the sensor and the reinforcement fibers, was considered to be constant; the resin's mass that surrounds the sensor is very small and it can be ignored.

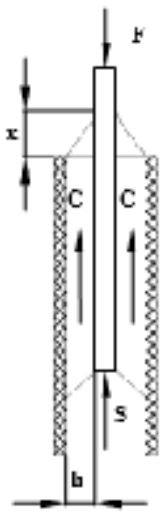

Fig. 2. The model of the studied system: $\mathrm{C}$-the damping coefficient of the resin; S -shear force; F-force;

$\mathrm{h}$-distance from sensor to fiber; $x$-the height of the resin ramped on the sensor

Considering these approximations, the system's equation of motion is:

$$
\begin{aligned}
& M \ddot{x}+C \dot{x}+K x=F \\
& F=F_{0} e^{i \omega t}
\end{aligned}
$$

where: $M$ is the system's mass that includes the sensor as well as its fastening system to the movement source, including the mass of the electrodynamic excitation system; $C$ the resin's viscous damping coefficient; $K$ elastic constant; $x$ height of the increased resin on the sensor.

$$
\text { Noting: } K x=2 S \text {, şi } 2 S=\frac{2 A G}{h} x=G^{*} x
$$

where: $S$ is the shearing force on one surface of the sensor; $G$ is the apparently shearing module in the plan; $A$ is the surface area of contact between the sensor and the resin.

Then the equation (5) becomes:

$$
M \ddot{x}(t)+C \dot{x}(t)+G^{*} x=F_{0} e^{i \omega t}
$$

Applying the Fourier transform to the equation (8) according to the equation (1), the response function in the frequency $H(\omega)$ is given by the mathematical relation:

$$
H(\omega)=\frac{\ddot{X}(\omega)}{F(\omega)}=\frac{\omega^{2}}{\sqrt{\left(G^{*}-M \omega^{2}\right)^{2}+(C \omega)^{2}}}
$$

where: $F(\omega)$ is the excitatory force measured with the piezoelectric force transducer; $\ddot{X}(\omega)$ is the system's acceleration measured with the piezoelectric accelerometer.

At the system's resonant frequency it can be written:

$$
G^{*}-M \omega^{2}=0
$$

Consequently, with the help of the equation (10) it can be determined after the experiments the complex shearing modulus.

\section{Results and discussions}

Virtual instrument for the determination of the sensor's oscillation frequency

For determining the shearing module of the composite polymeric materials a virtual instrument was designed with the help of LabVIEW's program [7, 8]. The virtual instrument can monitor in real time the processing of the composite materials and implicitly it can bring a dynamic mechanical analysis [9]. The instrument filtrates and processes with a weighting function of Hanning type of the excitatory signal as well as the response signal of the sensor placed in the studied composite material. There is the possibility to obtain the response in the composite system's frequency and to determine the frequency's peak, using the sweeping method in the fixed frequency domain of the exciting signal of the sensor inside the material. The frequency's spectrum was registered during the whole processing of the composite material at different temperatures with the help of a data acquisition card.

This virtual instrument was designed in order to include three components: a signal generator which can generate four types of drive signals from theelectrodynamic excitation system; spectrum analyzer which can calculate the average RMS of the power's spectrum of the acquisited signal detecting the resonant frequency's peak and the power's peak ; the oscilloscope with two channels which acquire and display simultaneously the waves' form of two signals: the exciting signal and the response signal of the tested composite structure.

The frontal panel of the virtual instrument is presented in figure 3 and in figure 4 it is presented the block diagram.

The advantages of this virtual instrument is that it can realize a dynamic mechanical analysis of the studied composite material in real time and that it can display the results of the spectral analysis with the same speed with which the analogical data is received from the piezoelectric force transducer to the piezoelectric accelerometer, realizing the processing in minimum time of the whole quantity of information contained in the analogical analized signals.

\section{Determining the shearing modulus}

To be able to conduct the experiments it was created a stand which allows the properties of the viscoelastic polymeric resins to be measured in real time and also the determination of the shearing modulus of the studied 


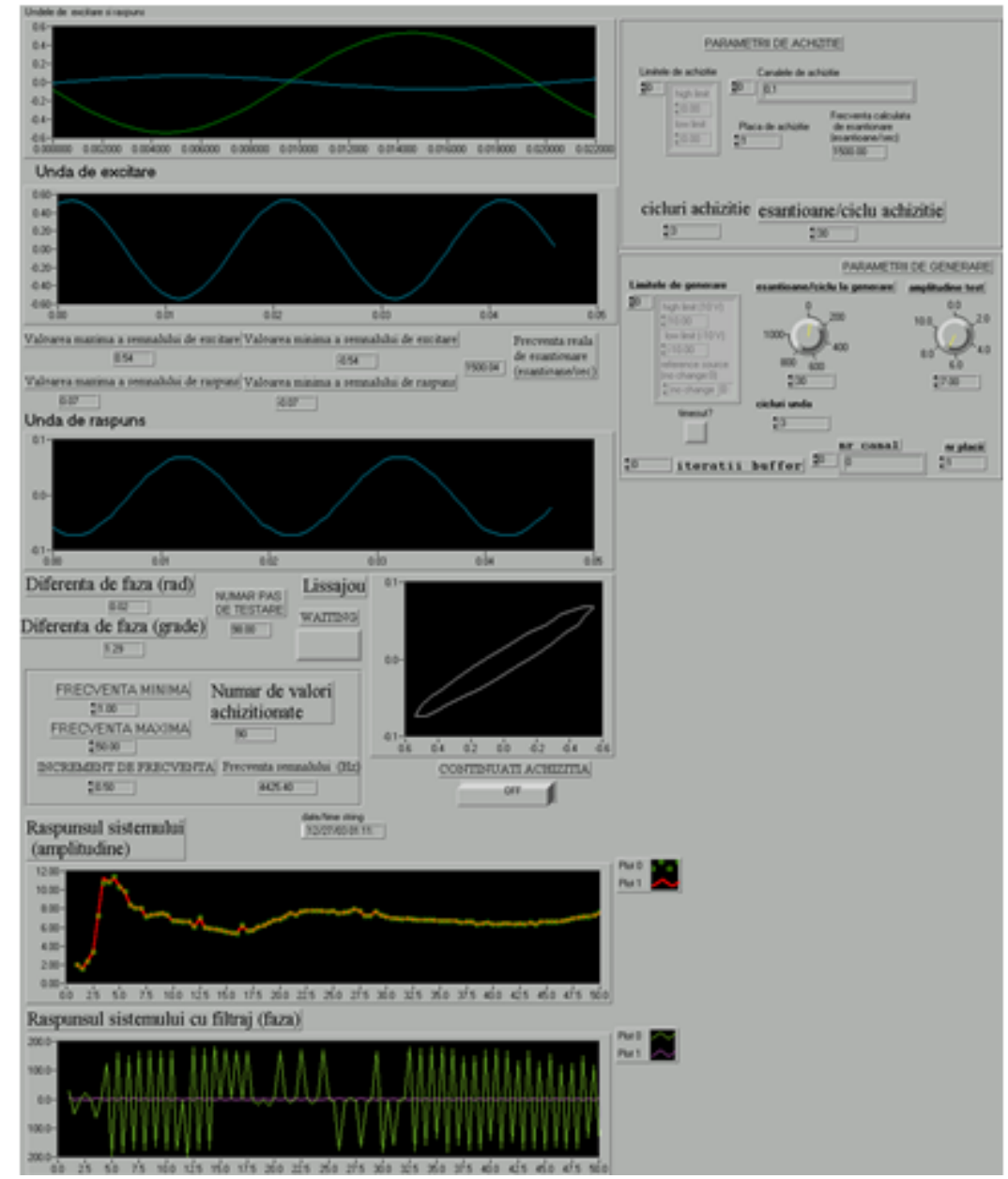

Fig. 3.The frontal panel of the virtual instrument

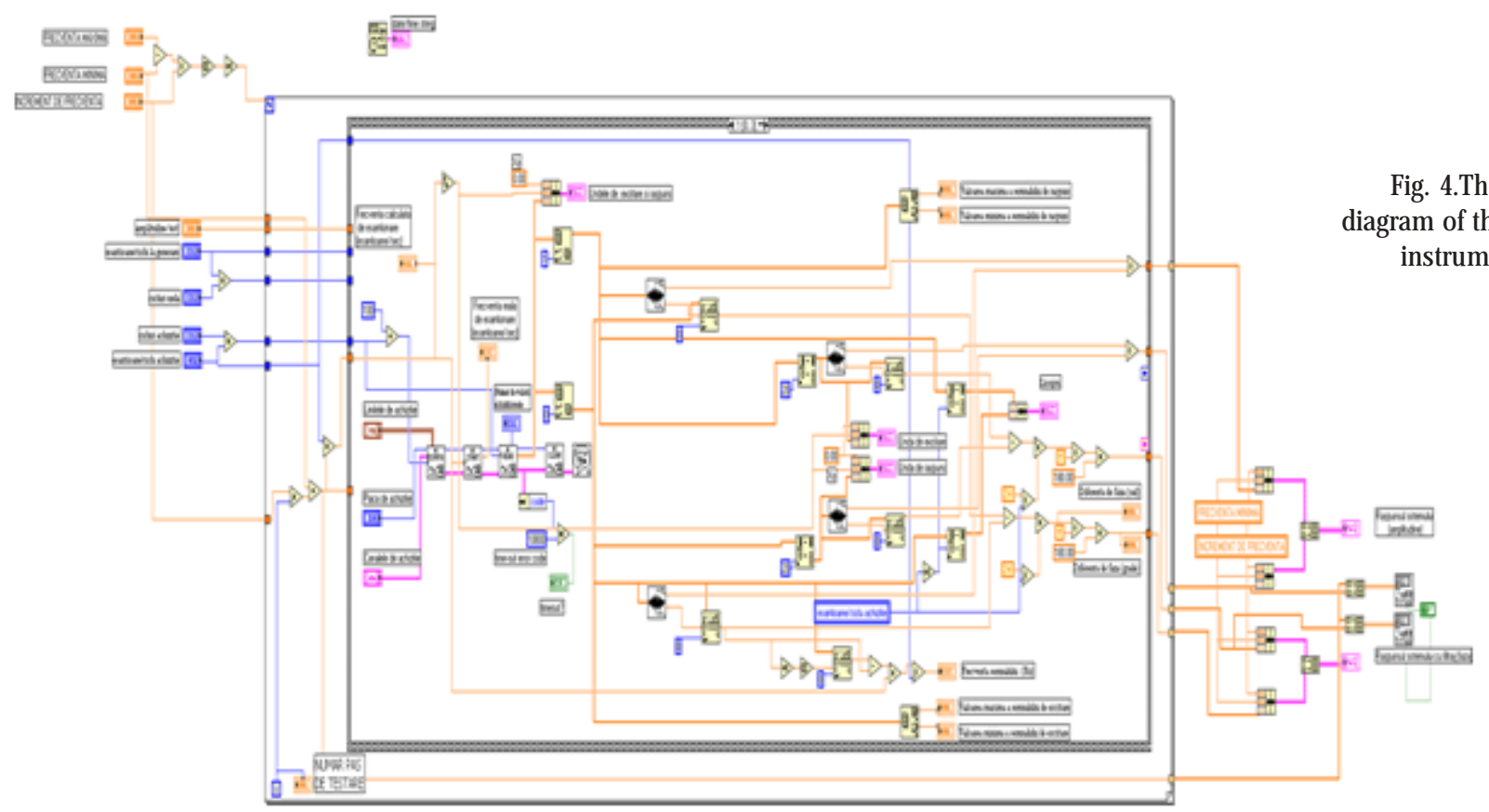

composite materials. The concept scheme of the stand is presented in figure 5.

The stand was created based on the concept of a sensor of whose operating is based on the analysis of mechanical impedance. Realizing this experimental stand led to allowing measuring in real time the viscoelastic properties of the polymeric thermo resistant resins and of the composite materials with such a matrix, determining the dynamic mechanical characteristics of the studied composite structure and finally determining the degree of ability to procession of the composite material $[9,10]$.
The analysis method of mechanical impedance supposes in fact a quantitative measurement of the effect of an exciting force in a structure. The report between the movement created by the exciting force and the force or in reverse in the frequency domain is called the response function in frequency or the transfer function $H(\omega)$, which is a parameter that implicitly contains the dynamical mechanical properties of the material. Consequently the analysis of the mechanical impedance of a vibrating probe that moves inside the resin or inside a composite material can be used for obtaining the shearing complex modulus of the resin and respectively of the composite system. At 


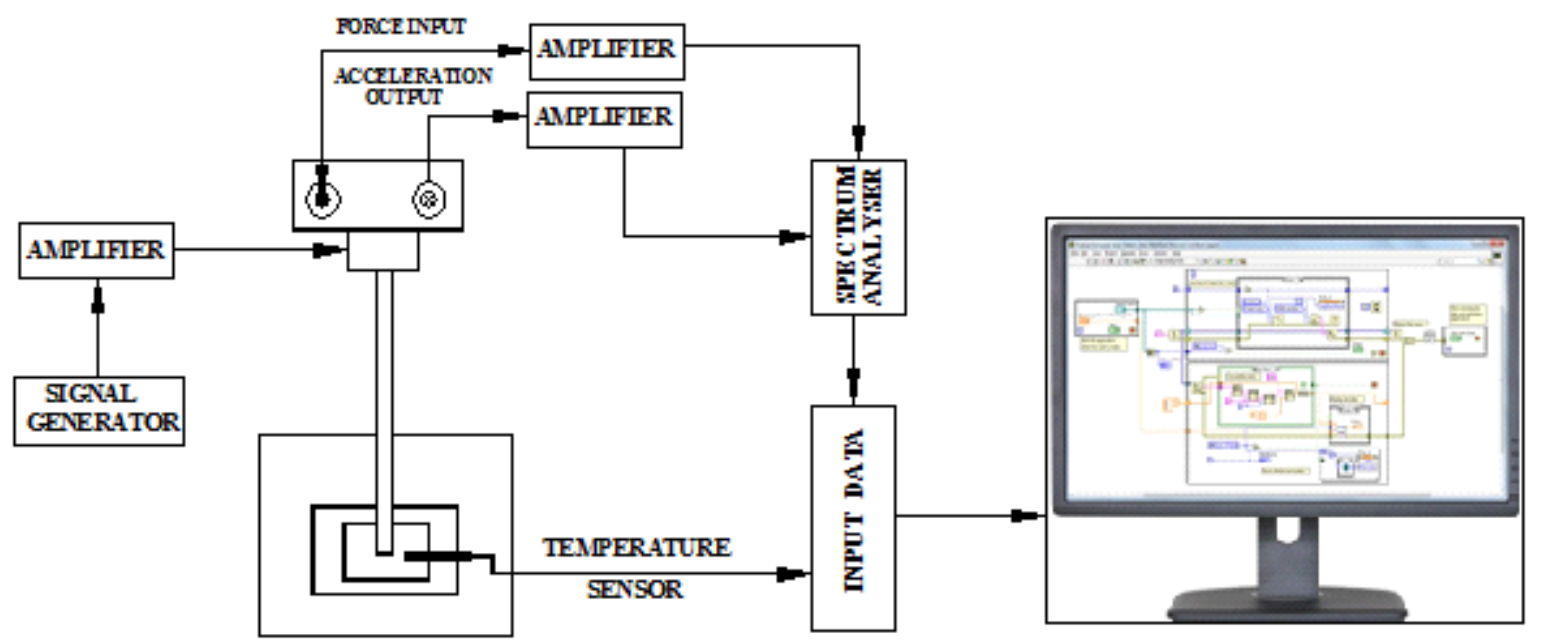

Fig. 5. Experimental stand

the interface between the sensor and the studied system a shearing resonant movement is created due to the movement of the sensor in the resin or between the composite material's layers under the action of the excitatory oscillated signal. The element in motion is in this case the sensor inside the composite structure. By measuring the absolute peaks of the moving probe in the composite material and the size of the exciting force at resonance approximations could be made about the variation of the shearing complex module $G^{*}$ and the viscous damping coefficient $C$. Considering all these aspects at the basis of the experimental research and the construction of the stand stayed in fact a problem regarding vibrations.

All the experiments were conducted following the same procedure: the sensor's action was realized with a exciting sinusoidal signal of a constant amplitude $5 \mathrm{~V}$ generated with the help of the virtual instrument. The machinery was programmed in order to automatically realize the sweep of the frequency domain $0 \ldots .20 \mathrm{~Hz}$. Both signals (the exciting and the response ones) were amplified with a charge amplifier. The form of the exciting waves and the response waves obtained as well as the phase angle between these can be seen on the frontal panel of the machinery. The two amplified signals were inserted in a spectral analyzer FFT including as well the virtual instrument. The determination of the resonant frequency of the system for the fixed range of frequencies was trying to be achieved and also obtaining the response function in the frequency $H(\omega)$, as a report between the Fourier transform of the response and exciting signals. The frequency's spectrum was registered on the whole period of the processing of the composite material at the environmental temperature and also at $50^{\circ} \mathrm{C}$.

To be able to conduct the experiments there were used samples of composite layered material at [45/45] $]_{20}$ and $[90 / 90]_{32}$ treated at the environmental temperature and also $50^{\circ} \mathrm{C}$. The matrix of the composite material from which the samples were made is composed of an epoxy resin of the DGEBA type - Epilox T19-36 and the H10-30 hardener. The hardener is a modified cycloaliphatic polyamine, which allows to increase the quality of the obtained products. The reinforcing material used was E-glass fiber fabric with continuous filaments.

Samples of composite material were obtained in a removable aluminum die shown in figure 6 .

Samples of composite material were created by using 20 layers of fiberglass with the topology [45/45] and [90/ ${ }_{90}{ }_{20^{\prime}}$ cured at ambient temperature and at $50^{\circ} \mathrm{C}$ (figs. 7 $\left.{ }^{10}\right)^{20^{\prime}}$. In all the experiments on this material both the

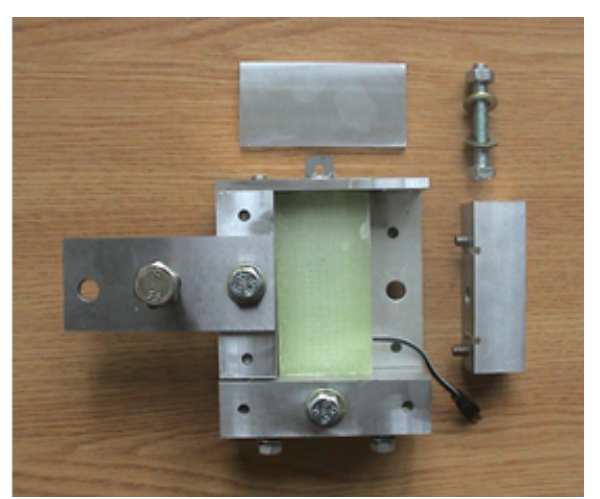

Fig. 6. Die used for processing composite material samples

temperature sensor and the vibrating sensor were put in the middle of the material between the $10^{\text {th }}$ and $11^{\text {th }}$ layers.

In the curing process of the composite materials with polymeric matrix, the dynamic mechanical properties were controlled with the help of: the temperature $T$ and the cross-linking degree $\alpha$ [11]. If the temperature increases, the viscosity and the stiffness of the polymeric matrix decrease. The cross-linking degree of the composite material grows with the temperature's grow th and treating time leading to an increased viscosity and stiffness [12].

From the acquisition in real time of the frequency it was determined the shearing modulus for the test-pieces from the composite material with the topology [45/45] $]_{20}$ and [90/90], at the environmental temperature and al so af $50^{\circ} \mathrm{C}$.

In the figure 11 it is presented the range in time of the shearing modulus for the specimens with the topology $[45 / 45]_{20}$.

In the figure 12 it is presented the range in time of the shearing modulus for the specimens with the topology $[90 / 90]_{20}$ :

From the analysis of the range of the sharing module, no matter the topology, it can be observed that the treating temperature in the two cases does not influence in a great manner the final shearing modulus but the moment of producing maximum heat from the polymerisation reaction is different [13-15]. At the environmental temperature the gelling time exceeds 50 min at both studied topologies and at $50^{\circ} \mathrm{C}$ the time is significantly reduced, reaching 30 40 minutes.

In figures 13 and 14 there are presented the graphs of variation in time of the shearing modulus $G$ at the environmental temperature and respectively at $50^{\circ} \mathrm{C}$ for both topologies [45/45] $]_{20}$ and $[90 / 90]_{20}$.

The influence of the topology of the studied composite material can be observed in figures 13 and 14 . For both treating temperatures of the composite material, the samples with the topology [45/45] ${ }_{20}$ had a bigger value of 


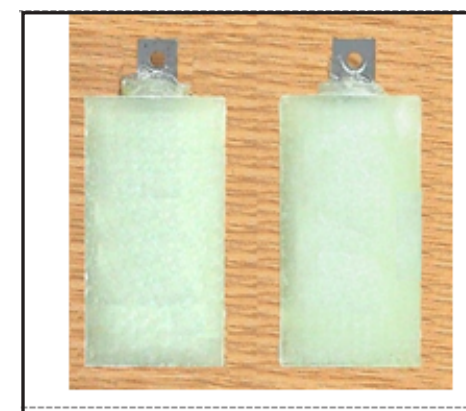

Fig. 7. Samples with the topology $[90 / 90]_{20}$ cured at $25^{\circ} \mathrm{C}$

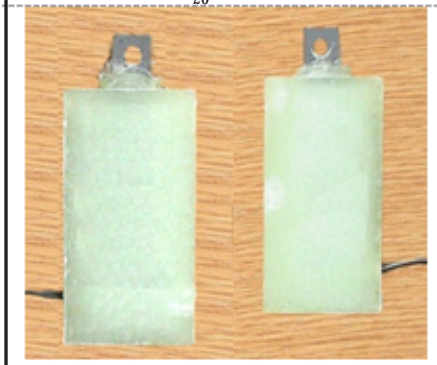

Fig. 9. Samples with the topology $[90 / 90]_{20}$ cured at $50^{\circ} \mathrm{C}$

the shearing modulus from the ones with the topology [90/ $\left.{ }^{90}\right]_{20}$. In the case of the treated samples at the environmental temperature the shearing modulus of the composite material with the topology $[45 / 45]_{20}$ is 4.08 $\mathrm{MPa}$, and the composite material with the topology [90/ 90] is $3.58 \mathrm{MPa}$. The same decrease of approximately $12.5 \%$ was noticed in the case of the composite materials treated at $50^{\circ} \mathrm{C}$. At this temperature the shearing modulus of the composite material with the topology $[45 / 45]_{20}$ is
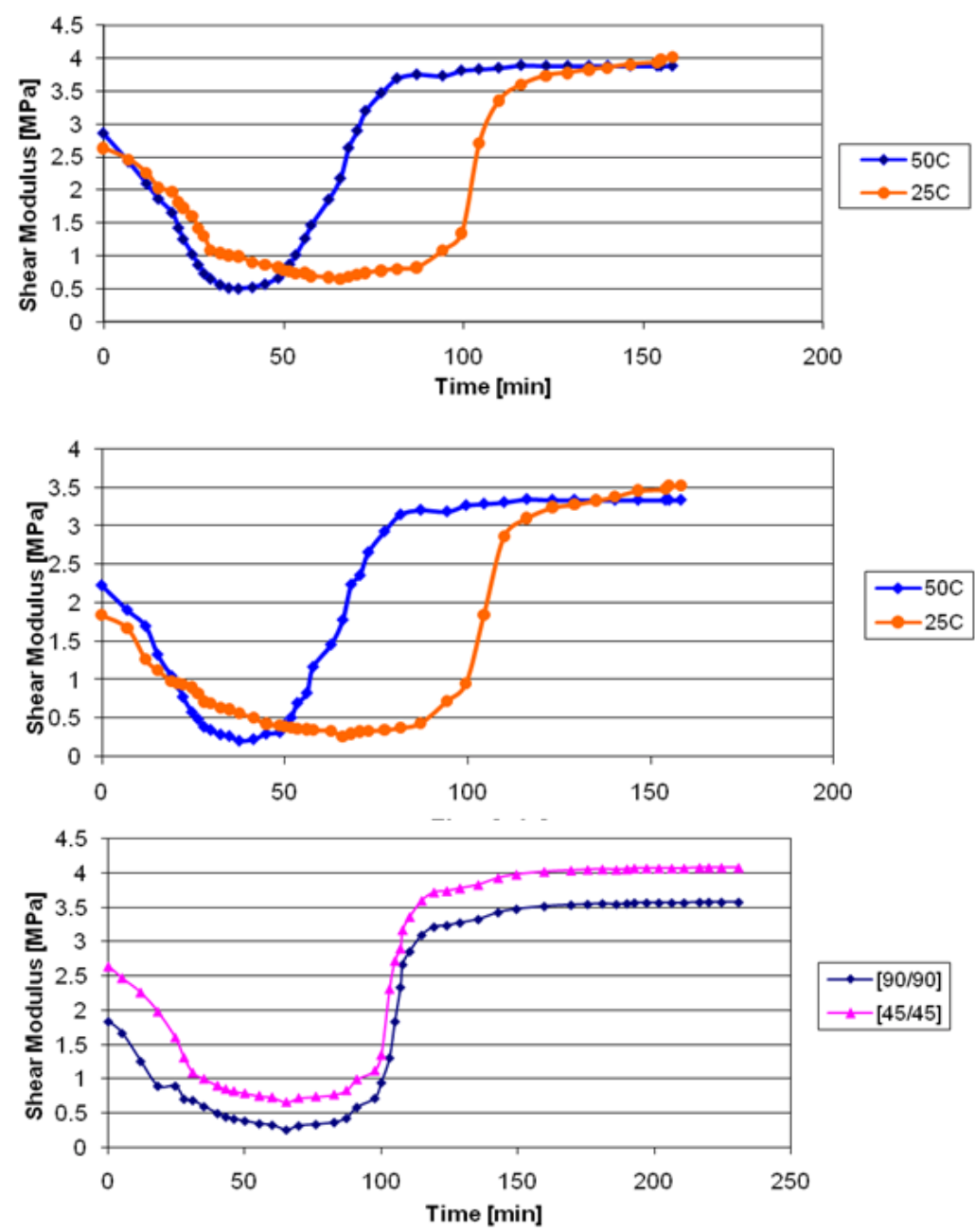

Fig. 11. Time variation of shear modulus for $[45 / 45]_{20}$ topology sample

Fig. 12. Time variation of shear modulus for $[90 / 90]_{20}$ topology samples

Fig. 13. Time variation of shear modulus for specimens with [45/45] $]_{20}$ and $[90 / 90]_{20}$ topology samples cured at ambient temperature 

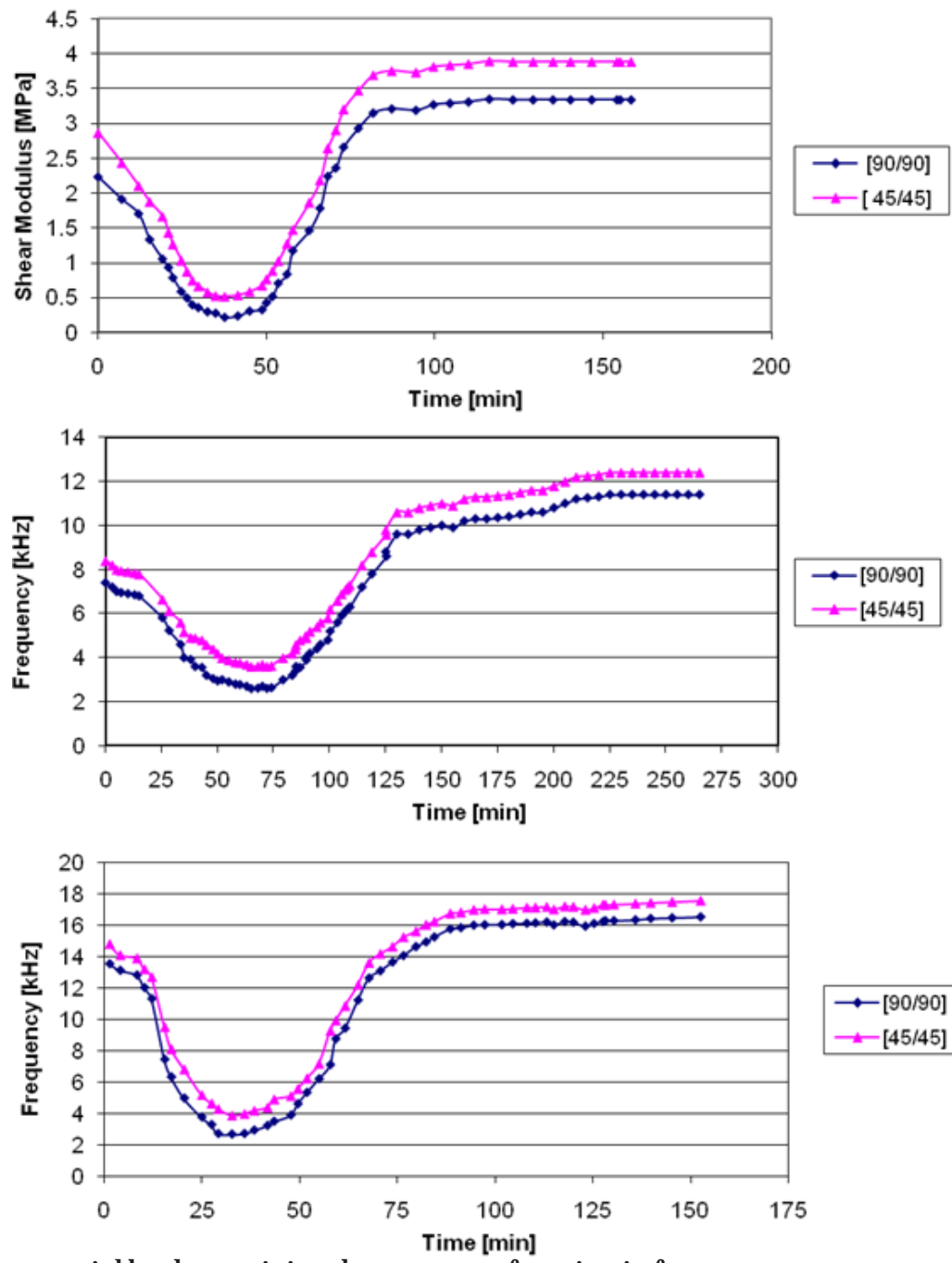

material by determining the response function in frequency as a report between the exciting force and the system's response. The graphs with the variation in time of the exciting frequency are presented in the figures 15 and 16.

Analyzing the graphs aforementioned itcan be observed that with the increasing treating temperature of the composite material the minimum of the exciting frequency moves towards the origin from the interval of 50-75 min to the interval of 25-50 min, meaning rushing the gelling point of the composite material.

\section{Conclusions}

In this article there are presented the experimental results obtained after monitoring the processing of a polymeric composite material. The purpose was characterizing the treating state of the composite material as well as determining the dynamic mechanical characteristics of the composite material and respectively the shearing modulus. For characterizing the treating state of the composite material it was used a sensor based on the analysis of the mechanical impedance. The functioning of the system is based on the determination of the relation between the exciting force of the sensor as input and the response of the sensor to the excitation as output.

The experimental results presented in this article show that this type of sensor based on the analysis of mechanical impedance can be used in detecting the viscoelastic transitions resulted from the treatment of the composite materials with a matrix of thermo resistant resins, being able to detect the changes of the shearing modulus apparently accompanied by the gelling and vitrification of the resin. Modifying the value of the shearing modulus during
Fig. 14. Time variation of shear modulus for specimens with topology $[45 / 45]_{20}$ and $[90 / 90]_{20}$ cured at $50^{\circ} \mathrm{C}$

Fig. 15. Variation of the excitation frequency for specimens with topology $[45 / 45]_{20}$ and $[90 / 90]_{20}$ cured at ambient temperature

Fig. 16. Variation of the excitation frequency for specimens with topology [45/45] ${ }_{20}$ and [90/90 $]_{20}$ cured at $50^{\circ} \mathrm{C}$

the treatment is related with the rheological and thermo dynamic changes in the material.

Regardless the topology of the composite material it was noticed that the treating temperature does not influence in a great way the final value of the shearing modulus. Instead the moment of maximum heat of the polymerisation produced is different.

The virtual instrument created realized the precise determination of the minimum exciting frequency appropriate with the starting point of the gelling process of the composite material. This method of analyzing the mechanical impedance can be applied successfully in monitoring the cross-linking process of the composite materials with polymeric matrix. The method, supported by all the machinery on whose base was developed can be used to characterize in real time the cross-linking state of the polymeric composite materials. Furthermore it can be used to produce the polymeric composite materials ensuring reliable and reproducible products.

The technique proposed in this article having the basis of a virtual instrument with high adaptability can be used in determining the dynamic mechanical properties of the polymeric composite materials in different curing stages.

\section{References}

1.MALKIN, A. YA. and KULICHIKHIN, S.G., Rheokinetics of Curing, Advances in Polymer Science, 1991, pp. 217-257.

2.MACOSKO, C. W., Rheological Changes During Crosslinking, British Polymer J ournal, 1985, 17, no. 2, pp. 239-245.

3.TUNG, C.M. and DYNES, P.J., Between Viscoelastic Properties and Gelationin Thermosetting Resins, J ournal of Applied Polymer Science, 27, 1982, pp. 569-574. 
4. STAN, F., MUNTEANU, A. V., FETECÃU, C., Analysis of Visco-elasticplastic Behaviour of Short Glass Fiber-reinforced Polyamide 66 Composite (PA66 GF30), Rev. Mate. Plast., 48, no. 1, 2011, p. 1-6.

5.ELLIS, B., The kinetics of cure and network formation, Chemistry and Technology of Epoxy Resins, ed., Blackie Academic and Professional, New York, 1993, pp. 72-116.

6.ARPITHA, G. R., SANJ AY, M R., YOGESHA, B., Review on Comparative Evaluation of Fiber Reinforced Polymer Matrix Composites. Carbon 4000, 30, 2014.

7.***LabVIEW - Graphical Programming for Instrumentation, National Instruments, 1996.

8.MUNTEEAU, M., LOGOFATU, B., Instrumentape virtuala - Labview, Editura Credis 2003.

9.SPANU, P., AMZA, C. Gh., DINU, G., Statistical Analysis of Some Mechanical Characteristics of Glass Fiber Reinforced Composites, Mat. Plast., 54, no. 4, 2017, p. 601-605.

10.STARK, W., GOERING, H., MICHEL, U., BAYERL, H., Online monitoring of thermoset postcuring by dynamic mechanical thermal analysis DMTA, Polym. Test. 2009, 28, pp. 561-566.
11.MIN LI, QI ZHU, PHILIPPE H. GEUBELLE and CHARLES L. TUCKER III, Optimal Curing for Thermoset Matrix Composites: Thermochemical Considerations, Polymer Composites, 22(1), 2001, pp. 118-131.

12.ZHU, L., PITCHUMANI, R., Analysis of a process for curing composites by the use of embedded resistive heating elements, Comp. Sci. Tech. 60, 2000, pp. 2699-2712.

13.0'BRIEN, D.J., MATHER P.T., WHITE S.R., Viscoelastic properties of an epoxy resin during cure, Journal of Composite Materials, 2001, 35(10), pp. 883-904.

14.SERBAN, D. A., VOICONI, T., LINUL, E., MARSAVINA, L., MODLER, N., Viscoelastic Properties of Pur Foams Impact excitation and dynamic mechanical analysis, Mat. Plast., 52, no. 4, 2015, p. 537-541.

15.THORPE, R., POURSARTIP, A., Experimentally determining the viscoelastic behavior of a curing thermoset epoxy, 19th International Conference on Composite Materials, Montreal, 2013, pp. 1997-2002.

Manuscript received: 23.09 .2018 